\title{
Signatures of long-lived gluinos in split supersymmetry
}

\author{
JoAnne L. Hewett ${ }^{a}$, Ben Lillie ${ }^{a}$, Manuel Masip ${ }^{a, b}$ and Thomas G. Rizzo ${ }^{a *}$ \\ ${ }^{a}$ Stanford Linear Accelerator Center, 2575 Sand Hill Rd. Menlo Park, CA 94025 \\ ${ }^{b}$ CAFPE and Departamento de Física Teórica y del Cosmos \\ Universidad de Granada, 18071, Granada, Spain \\ E-mail: hewett@slac.stanford.edu, lillieb@slac.stanford.edu, \\ masip@slac.stanford.edu, rizzo@slac.stanford.edu
}

Abstract: We examine the experimental signatures for the production of gluinos at colliders and in cosmic rays within the split supersymmetry scenario. Unlike in the MSSM, the gluinos in this model are relatively long-lived due to the large value of the squark masses which mediate their decay. Searches at colliders are found to be sensitive to the nature of gluino fragmentation as well as the gluino-hadron interactions with nuclei and energy deposition as it traverses the detector. We find that the worst-case scenario, where a neutral gluino-hadron passes through the detector with little energy deposition, is well described by a monojet signature. For this case, using Run I data we obtain a bound of $m_{\tilde{g}}>170 \mathrm{GeV}$; this will increase to 210(1100) GeV at Run II(LHC) if no excess events are observed. In the opposite case, where a charged gluino-hadron travels through the detector, a significantly greater reach is obtained via stable charged particle search techniques. We also examine the production of gluino pairs in the atmosphere by cosmic rays and show they are potentially observable at IceCube; this would provide a cross-check for observations at hadron colliders.

Keywords: Beyond the Standard Model, Supersymmetric Standard Model, Supersymmetry Phenomenology.

*Work supported by the Department of Energy Contract DE-AC02-76SF00515. M.M. also supported by a grand from the MECYD (PR2003-0466), by MCYT (FPA2003-09298) and Junta de Andalucía (FQM-101). 


\section{Introduction}

The Minimal Supersymmetric Standard Model (MSSM) enjoys considerable phenomenological success, including the generation of electroweak symmetry breaking, the prediction of grand unification, and the presence of a dark matter candidate [1]. However, the existence of naturally large supersymmetric contributions to flavor changing neutral currents [2], to electric dipole moments (edm) [3, 4], and to proton decay are well-known and have long plagued supersymmetric model builders. In addition, the lack of observation of a light Higgs boson at LEP II [5] has created tension in the MSSM parameter space and indicates the existence of tuning at the level of at least a few percent. These outstanding issues have prompted a continuing vast array of research over the last two decades, and numerous candidate supersymmetric models have been put forward.

Recently, a supersymmetric model has been proposed $[6,7]$ which retains the successes of supersymmetry, solves the flavor and CP problems, and extends the proton lifetime, albeit at the cost of naturalness. This model, known as Split Supersymmetry, postulates that Supersymmetry breaking occurs at the very high scale, $m_{S} \gg 1000 \mathrm{TeV}$. The scalar particles all acquire masses at this high scale, except for a single neutral Higgs boson, whose mass is either finely-tuned or is preserved by some other mechanism. The fermions in the theory, including the gauginos, are assumed to be protected by chiral symmetries and thus can have weak-scale masses. (For other supersymmetric models with heavy scalars and weakscale fermions, see [8].) The existence of weak-scale fermions $(i)$ preserves gauge coupling unification even if the scalar partners are ultra-heavy since only complete $S U(5)$ multiplets are being removed from the low energy spectrum, and (ii) provides a natural dark matter candidate in the lightest neutralino. The ultra-heavy masses for the scalars guarantee the absence of large supersymmetric flavor changing interactions, since all such processes are mediated by the sfermions at loop level if R-parity is conserved (or at tree-level if R-parity is violated). The generic constraints from flavor and edm data which set $m_{S}>100 \mathrm{TeV}$ and $1000 \mathrm{TeV}$, respectively, are easily satisfied within this model. Likewise, the familiar dimension-five operator which mediates proton decay, $q q \tilde{q} \tilde{\ell}$, is also suppressed, delaying proton decay which now occurs via dimension-six operators. This increase in the proton lifetime is also in agreement with data[9]. Since the theory is not supersymmetric below the scale $m_{S}$, the usual relations between the supersymmetric gauge and Higgs sector (i.e., between the Yukawa, quartic and gauge couplings) no longer hold. In particular, Renormalization Group Evolution in this model yields a prediction [10] for the lightest Higgs mass of $m_{h}=130-170 \mathrm{GeV}$; this differs from that of the MSSM and is in agreement with the null searches at LEPII. However, since the value of the sfermion masses are ultra-heavy, the large quadratic corrections to the mass of the Higgs are not cancelled in the manner present in weak-scale supersymmetry, and the Higgs sector remains extremely fine-tuned. Split Supersymmetry proponents argue that this tuning may, indeed, be present in nature and may be related to the cosmological constant problem which suffers an even greater degree of fine-tuning. For example, it is possible that the tuning in the Higgs mass may be consistent with the solution to the cosmological constant 
problem based on the string theory landscape.

Whether or not one takes the motivation for Split Supersymmetry seriously, it is important to consider the phenomenological implications of having such a fine-tuning present in nature. One generic feature of this model is that the gluino is very long-lived, since the squarks which mediate its decay are ultra-heavy. It could easily appear to be stable in collider processes. In this paper, we will examine the signatures of a long-lived gluino in both collider and cosmic ray detectors. Collider signals for such particles have been previously examined in $[11,12,13]$, and are found to be dependent on the charge of the gluino-hadron resulting from fragmentation which dictates the amount of energy deposition by the gluino hadron as it traverses the detector. Here, we point out the importance of the monojet signature, arising from gluino pair production plus a jet bremsstrahlung with the gluinos escaping the detector unobserved; this signal is present for all possible charges of the gluino-hadron. We also consider the charged gluino-hadron constraints from charged stable particles searches. In both cases, we find constraints from current data from $p \bar{p}$ collisions at the Tevatron Run I, and then estimate the search reach of Run II and the LHC. In addition, we examine the signals in very large neutrino detectors, such as IceCube, from the production of gluino pairs in the atmosphere by cosmic rays. All our results are essentially independent of the Split Supersymmetry model details and are applicable to any model which contains a stable or meta-stable heavy colored particle.

This paper is organized as follows. In section 2 we briefly review Split Supersymmetry, and the properties of the gluino in this proposal. In section 3 we show how constraints can be placed on the mass of the gluino from present Tevatron data, and what the search reaches might be at Run II and the LHC. In section 4 we describe a possible signal in the IceCube detector from the cosmic ray production of gluinos. Section 5 concludes.

\section{Phenomenological Features of Split Supersymmetry and Long-Lived Gluinos}

The rationale behind Split Supersymmetry has been discussed in the previous section. The essential phenomenological ingredients are that supersymmetry breaking occurs near the GUT scale with $m_{S} \gg 10^{6} \mathrm{TeV}$ and all scalar masses, except for a single finely-tuned light Higgs, are set to that scale. Whereas the fermions are protected by a chiral symmetry and have masses at the weak scale. A feature of this supersymmetric mass spectrum is the dramatic reduction over the MSSM in the number of parameters at the $\mathrm{TeV}$ scale. In particular, there are only 9 new parameters relevant at TeV energies. These are the three gaugino masses, a $\mu$ term for the Higgsinos, the four Higgsino-Gaugino couplings, and the scale of the scalar masses and supersymmetry breaking, $m_{S}$. Many observables will only depend on the gaugino masses and $m_{S}$, yielding predictions which are robust with respect to variation over the parameter space. We also note that since supersymmetry is not present at the $\mathrm{TeV}$ scale, the weak-scale

Higgsino-Gaugino couplings differ from their usual MSSM values; these couplings approach these values as one evolves towards $m_{S}$. 
This scenario produces a strikingly different phenomenology from that of the MSSM. Some features have been examined in recent papers, including the implications for dark matter detection [7, 14], the Renormalization Group running from the high scale $m_{S}[10]$, and aspects of sparticle detection at colliders $[7,15,16]$.

The absence of the scalars at the $\mathrm{TeV}$ scale affects both the production and decays of the gauginos. Although we will focus on the gluinos in this paper, we first make a few comments on the electroweak gaugino sector. In the MSSM, most sparticles decay through a cascade down to the lightest supersymmetric particle (LSP), and these cascade decays usually involve sfermions at some stage. Due to the heavy scalar masses in Split Supersymmetry, these cascade decays will essentially not occur. This has a two-fold effect on the electroweak gaugino phenomenology: ( $i$ ) Cascade decays of heavier sparticles provide the main production mechanism for charginos and neutralinos at hadron colliders. Since the squarks are beyond the kinematic reach and, as we discuss at length below, the gluino is long-lived, cascade decays of these particles are no longer a source of electroweak gaugino production. In addition, heavier gauginos are not likely to cascade. Hence the electroweak gauginos can only be produced via the Drell-Yan mechanism. (ii) The only decay channels open to the electroweak gauginos are direct decays to the electroweak gauge bosons, such as $\tilde{\chi}^{ \pm} \rightarrow W^{ \pm}+\tilde{\chi}_{1}^{0}$. The characteristic trilepton signature for electroweak gaugino production is still viable, but the lepton spectrums will be modified. Combined, these two features result in a lower production rate for charginos and neutralinos at hadron colliders and a more prompt lepton spectrum arising from their decays.

The lack of $\mathrm{TeV}$ scalars also affects the production and decay of the gluinos. The usual Feynman diagrams for gluino pair production via $g g$ and $q \bar{q}$ annihilation in the MSSM are displayed in Fig. 1. The fourth diagram proceeds by squark exchange and while it can make a significant contribution to the production cross section in the MSSM, it is negligible in the Split Supersymmetry model. The channels for gluino pair production in this scenario are thus the same as for heavy quark production in the Standard Model (SM). In addition, the associated production channel $q \bar{q} \rightarrow \tilde{g}+\chi_{i}^{0} / \chi_{i}^{ \pm}$is important in the MSSM as it can either contribute to the jets $+\mathbb{E}_{T}$ signal or produce leptons in the final state. This production proceeds via $\tilde{q}_{L, R}$ exchange in the $t$ - and $u$ - channels, and does not occur in the Split Supersymmetry scenario. In summary, we see that the absence of $\mathrm{TeV}$ scalars results in production cross-sections for gluinos that are somewhat lower than those generally considered in the MSSM.

We note that searches at LEP for stable hadronizing gluinos have been carried out $[17,18]$. Both ALEPH and DELPHI have searched in the channel $e^{+} e^{-} \rightarrow q \bar{q} g \rightarrow q \bar{q} \tilde{g} \tilde{g}$, and place the constraint $m_{\tilde{g}}>26.9 \mathrm{GeV}$ at $95 \% \mathrm{CL}$. They have obtained stronger limits in the case of squark pair production with subsequent decay into long-lived gluinos, however, these channels do not occur in the present scenario.

A striking feature of the Split Supersymmetry model is the extremely long lifetime of the gluino. Unlike the electroweak gauginos, it does not have a decay channel that avoids sfermions. The gluino can only decay through a virtual squark to a $q \bar{q}\left(q^{\prime}\right)+\chi_{i}^{0}\left(\chi_{i}^{ \pm}\right)$. The large mass of the squarks then implies a long lifetime for the gluino. This lifetime is given 


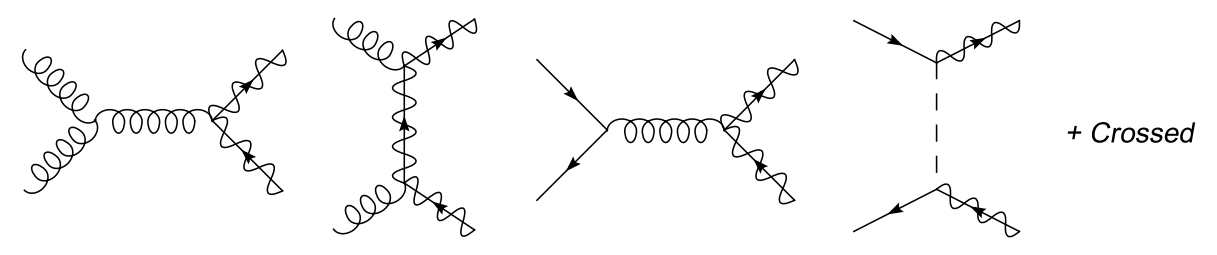

Figure 1: Diagrams contributing to gluino pair production in hadronic collisions in a generic supersymmetric model. The fourth diagram proceeds by squark exchange, and is effectively absent in Split Supersymmetry. The remaining diagrams are identical to those which mediate heavy flavor pair production.

approximately by (neglecting the mass of the electroweak gaugino)[19]

$$
\tau \simeq 8\left(\frac{m_{S}}{10^{9} \mathrm{GeV}}\right)^{4}\left(\frac{1 \mathrm{TeV}}{m_{\tilde{g}}}\right)^{5} \mathrm{~s}
$$

Here we have explicitly included the coupling constants and summed over the possible final states. Since the scale $m_{S}$ ranges from roughly $10^{7} \mathrm{GeV}$ up to the GUT scale (although $m_{S}<10^{12-13}$ is favored for cosmological reasons [6]), the gluino lifetime can easily vary from picoseconds to the age of the universe for gluino masses near the electroweak scale. If the lifetime is too long, then there may be cosmological problems from the relic density of gluinos. There is a strong limit on the density of heavy isotopes [20], which implies that either the gluino lifetime must be smaller than the age of the universe, or there is some mechanism in the early universe that insures they have a vanishingly small relic density.

A long-lived, but unstable, gluino produces interesting signals in collider experiments. For lifetimes around 1ps the gluino will most likely decay inside a silicon vertex detector, and could be found in a search for multi-jets plus $\mathbb{E}_{T}$ with displaced vertices. For lifetimes between $\sim 1$ ps and $\sim 100 \mathrm{~ns}$, the gluino will decay in the bulk of the detector. However, the signals from this decay will most likely be lost in the background, or not pass the trigger. It may be possible to reconstruct them off-line if the event contained something else which passed the trigger. Generically, however, for the bulk of the parameter space, the lifetime is expected to be larger than $10^{-7} \mathrm{~s}$, and gluinos produced in colliders will decay outside of the detector. In this case they will appear to be effectively stable, and search strategies for heavy stable particles need to be employed [21].

When the gluino is produced, it will hadronize into a color singlet state, called an $R$ hadron (since it carries one unit of $R$-parity) $[22,23]$. For notational purposes, we will denote the hadron $\tilde{g} q \bar{q}$ as an $R$-meson, $\tilde{g} q q q$ as a $R$-baryon and $\tilde{g} g$ as a $R$-gluon. Search techniques for such particles will depend on the characteristics of the $R$-hadron, in particular its electric charge. We discuss each possibility in detail below. 


\section{Searches at Hadron Colliders}

\subsection{Propagation inside a medium}

Once produced, the long-lived gluino will hadronize into a color singlet state $R$. If neutral, $R$ will experience energy loss only through hadronic collisions as it propagates through matter, whereas a charged $R$ will also deposit energy in the form of ionization. Notice that there is also the possibility that $R$ can change its charge in each hadronic interaction, resulting in a "flipper" R-hadron that is alternately charged and neutral as it propagates through matter.

The fragmentation probabilities into neutral versus charged $\mathrm{R}$ states are extremely uncertain. However, there are two cases which favor fragmentation to a neutral $R$-hadron: (i) the probability of fragmentation to $\tilde{g} q q q$ is much smaller than to $\tilde{g} g$, and (ii) the mass difference between $\tilde{g} q \bar{q}$ and the neutral state $\tilde{g} g$ is larger than $m_{\pi}$ ( $R$-meson states would then decay quickly into $\tilde{g} g$ and the produced hadron would be effectively always neutral). In any other case one would naively expect similar probabilities for the hadronization into charged or neutral states.

The possibility of a charge exchange for a gluino hadron following hadronic scattering is more involved. In particular, the fact that $R$-meson (and $R$-gluon) states may convert into baryon $R$ hadrons $(e . g ., \tilde{g} d \bar{d}+u u d \rightarrow \tilde{g} u d d+u \bar{d})$ but not vice-versa [24] has been overlooked in previous analyses of long-lived gluinos $[11,13]$. This conversion would be favored by the lightness of the final pion, whereas processes that convert $R$-baryon into $R$-meson states are negligible. In any case, to cover all the possibilities we will consider the limits where $R$ is always neutral or always charged, and will also briefly discuss the flipper model proposed in [24].

As $R$ propagates, hadronic energy loss will be dominated by interactions with the nucleons

in the medium (rather than partons or the nucleus as a whole). Little is known about these interactions, however several models have been discussed in the literature $[11,12,13,24]$. The rate of energy loss will depend on the average amount of energy deposited in each interaction $(\langle\Delta E\rangle)$ and the average distance between interactions in that medium $\left(\lambda_{T}(R)\right)$. The hadron $R$ can be viewed as a non-interacting heavy gluino surrounded by a colored cloud of light constituents that are responsible for the interaction. This simple picture suggests that $\langle\Delta E\rangle$ is independent of the gluino mass and only depends on the speed $(\beta)$ of the hadron. In addition, only a small fraction of the energy of $R$ is relevant to the interaction. For example, it is expected that a $\tilde{g} q \bar{q}$ hadron with $E=400 \mathrm{GeV}$ and $m=200 \mathrm{GeV}$ would strike a nucleon as if it were a light meson of mass $0.6 \mathrm{GeV}$ with $E=1.2 \mathrm{GeV}$. Therefore, $R$ will lose a very small fraction of its energy in each interaction.

In the process $R N \rightarrow R X$, where $X$ is a system of one or several final particles and $N$ denotes the incident nucleon, the energy loss is given by

$$
\Delta E=\frac{m_{X}^{2}-m_{N}^{2}+|t|}{2 m_{N}}
$$




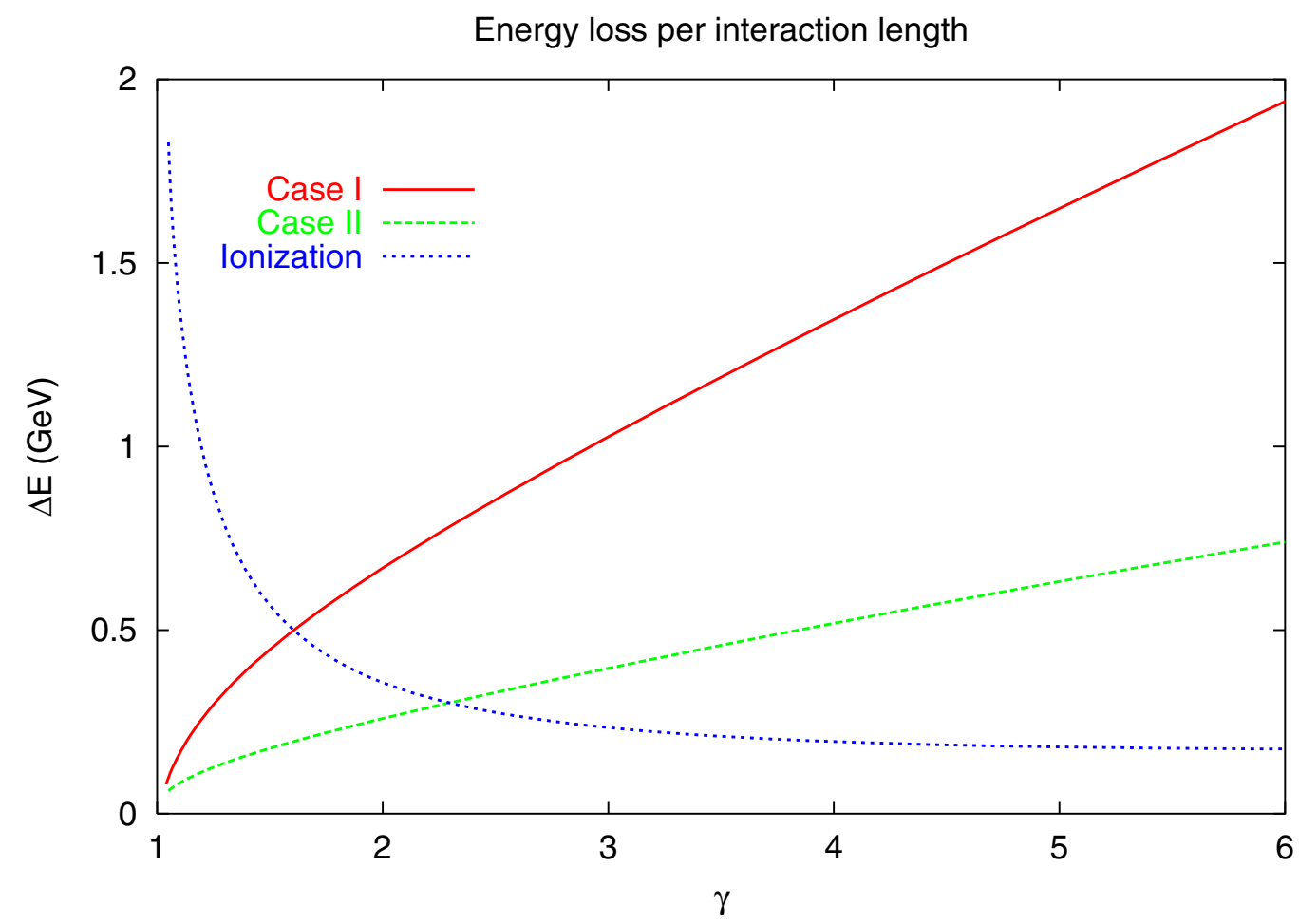

Figure 2: Energy loss by a $R$-hadron per interaction length due to hadronic interactions described by two models and due to electromagnetic ionization.

where the dominant values of the momentum transfer $|t|$ are of order $(0.1-1 \mathrm{GeV})^{2}$. We follow [11] and consider two different models describing the differential cross section $d \sigma / d|t| d m_{X}$ for this reaction: Case (1) the cross section is given by a constant differential for $|t|<1 \mathrm{GeV}^{2}$, and Case (2) it is given by a triple-Pomeron distribution described in [25]. It is remarkable that in both cases $\langle\Delta E\rangle$ can be well approximated by a linear expression when expressed in terms of $\gamma=\left(1-\beta^{2}\right)^{-1 / 2}$ (see Fig. 2). In particular, for $\gamma \geq 1.3$ we find $\langle\Delta E\rangle \approx k \gamma$, with $k=0.35 \mathrm{GeV}$ (case (1)) or $k=0.14 \mathrm{GeV}$ (case (2)). These results are consistent with those of [24], which also exhibit an approximate linear behavior, where when nuclear effects are included, it is found that $k$ lies between $0.3 \mathrm{GeV}$ for interactions in Iron and 0.2 $\mathrm{GeV}$ in Hydrogen. Since we are considering interactions inside a detector (as well as in the atmosphere in the following section), we therefore take cases (1) and (2) as upper and lower estimates for $\langle\Delta E\rangle$.

To estimate the mean interaction length of the $R$-hadron inside a collider detector, we follow [11] and take $\lambda_{T}(R)=(16 / 9) \lambda_{T}(\pi) \approx 19 \mathrm{~cm}$ in iron. The instrumented thickness of the calorimeter is around $8 \lambda_{T}(\pi)$ at CDF or $11 \lambda_{T}(\pi)$ at D0. For a neutral $R$ and $\lambda_{T}(R)=19$ $\mathrm{cm}$ this implies an average of 4.5 hadronic interactions depositing a total energy between $1.6 \gamma$ $\mathrm{GeV}$ (case (1)) and $0.63 \gamma \mathrm{GeV}$ in CDF. For a $R$-hadron mass of $100 \mathrm{GeV}$ with $E=400 \mathrm{GeV}$, this gives a total energy deposition up to $6.4 \mathrm{GeV}$. If $\lambda_{T}(R)$ is reduced by a factor of $1 / 2$ 


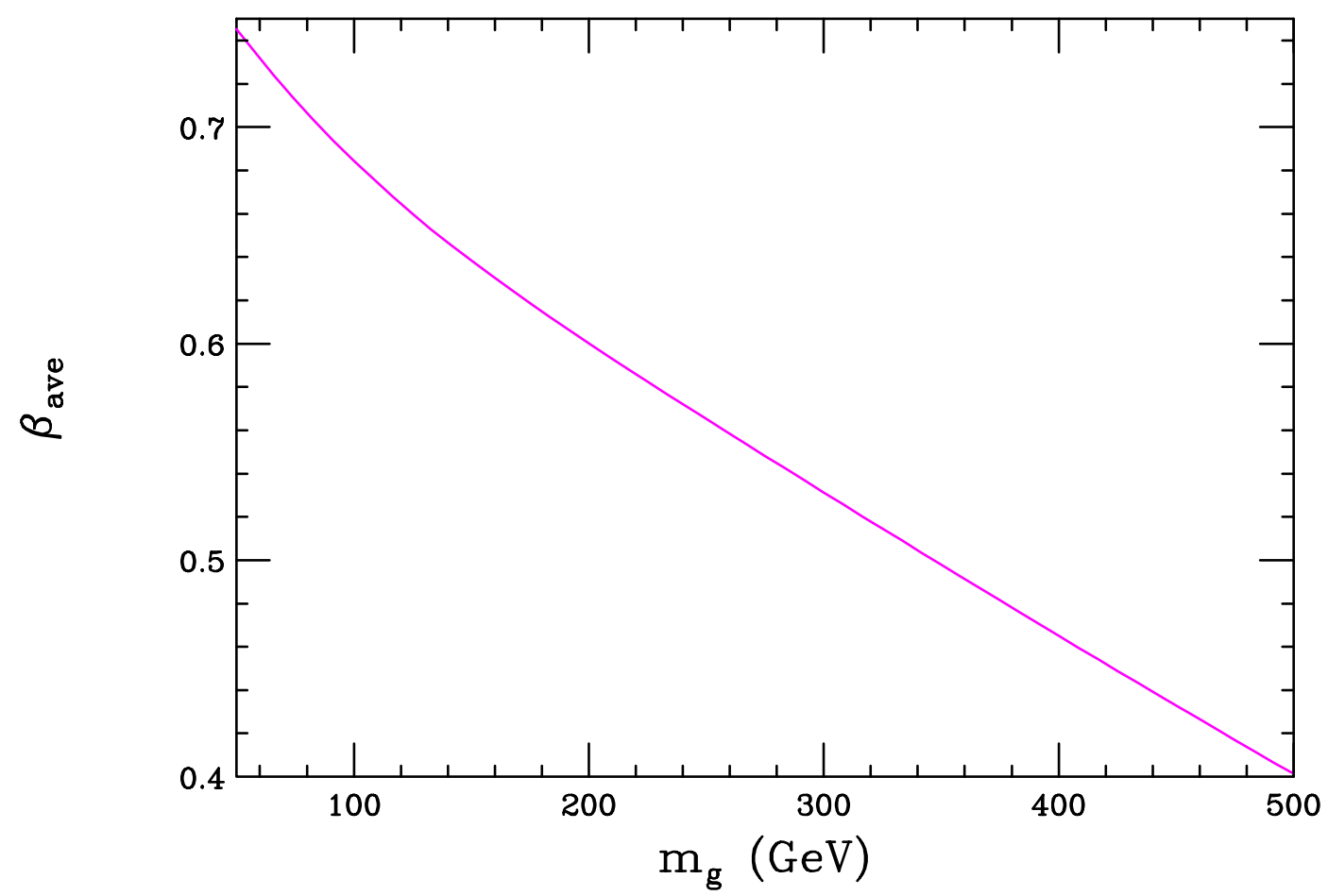

Figure 3: Average speed of a gluino produced at the Tevatron.

(which doubles the number of hadronic interactions in the calorimeter) the maximum energy loss visible at CDF would be just $12.4 \mathrm{GeV}$. Notice that if $m>100 \mathrm{GeV}$ or $E<400 \mathrm{GeV}$ (the typical values to be considered at the Tevatron) the total energy deposition would be even smaller.

For a charged $R$-hadron, the energy loss through ionization is described by the BetheBloch equation and becomes relevant at low values of $\beta$. Taking into account the $1 / \beta^{2}$ dependence, we find that energy deposition by ionization dominates for $\gamma \leq 1.5$ (see Fig. 2) and is much smaller than that for hadronic interactions when $\gamma \geq 2$. For a fast moving charged $R$-hadron (see Fig. 3 for the average speed of a gluino produced at the Tevatron), around $1.3 \mathrm{GeV}$ of ionization energy would be deposited in the CDF detector. Charged $R$ hadrons of mass $m \approx 100 \mathrm{GeV}$ produced with $\gamma \leq 1.1$ will lose all their kinetic energy (around $10 \mathrm{GeV}$ ) through ionization in the hadronic calorimeter.

In summary, fast $R$-hadrons lose energy mainly through hadronic interactions (their electric charge becomes irrelevant), whereas slow charged $R$-hadrons have sizeable energy loss only through ionization. In either case, the total amount of energy deposited in 1 meter of iron (the instrumented length in a hadronic calorimeter) will be smaller than $\approx 15 \mathrm{GeV}$ and may escape the experimental triggers. We note that the specific detector characteristics quoted here are for the Run I configurations; we assume they are roughly equivalent for Run II. 


\subsection{Neutral $R$-hadrons}

The most challenging situation in any scenario with a long-lived gluino is the case where the gluino fragments to form a neutral stable hadron which remains neutral as it traverses the entire detector. As discussed above, a neutral gluino hadron will have a mean interaction length of order $\sim 19 \mathrm{~cm}$ in the detector but will deposit only a few hundred MeV during each interaction. Given this small amount of energy deposition it is unlikely that the detector will be able to trigger on this signature without being swamped by soft QCD backgrounds. In fact, given the typical requirements for defining a jet at the Tevatron and LHC detectors, the pair production of gluinos fragmenting into neutral stable hadrons will appear as a rather soft process and is, hence, invisible. Thus, to trigger on events signaling gluino pair production something extra is required.

Here we will consider the production of a gluino pair in association with an additional jet with sufficiently high- $p_{T}$ so that it captures the attention of the trigger. Since the $R$ hadron energy deposition is rather soft, the gluino-hadron pair plus jet final state will be observed in the monojet channel with the neutral gluino hadrons appearing as missing energy. There are three subprocesses which contribute to this final state at leading order in QCD: $g g, q \bar{q} \rightarrow \tilde{g} \tilde{g}+g$ and $g q(\bar{q}) \rightarrow \tilde{g} \tilde{g}+q(\bar{q})$. In ordinary supersymmetry, squarks play an important role as intermediate states in these subprocesses. Here, as discussed above, in the limit where all the squark masses are ultra-heavy, the squarks formally decouple. The cross sections corresponding to these subprocesses are then found to be essentially the same as that for the $t \bar{t}+$ jet final state apart from the appropriate color factors. The matrix elements and kinematics required to calculate the relevant cross sections can be found in Refs. [26, 27] which we adapt for the present analysis.

The best current bounds on excess events in the monojet channel from the Tevatron are provided from searches for large extra dimensions by CDF from Run I [28] (see also [29]) with a data sample of $84 \mathrm{pb}^{-1}$. CDF requires at least one central jet with $E_{T} \geq 80 \mathrm{GeV}$ as well as missing transverse energy in excess of $80 \mathrm{GeV}$; if an additional jet is also present it must have $E_{T} \geq 30 \mathrm{GeV}$. To be called a jet, an energy cluster must have $E_{T} \geq 15 \mathrm{GeV}$ in this analysis so that the soft energy deposition arising from the neutral $R$-hadrons will not be seen as a jet. CDF observes 284 events passing their cuts while the SM Monte Carlo predicts $274 \pm 16$ from which a bound of 62 can be placed on the number of events arising from New Physics.

In Fig. 4 we show the cross section for gluino pairs in association with a jet at leading order for the Run I Tevatron $(\sqrt{s}=1.8 \mathrm{TeV})$ as a function of the gluino mass employing the CDF cuts. Since the calculation is only performed at leading order, the renormalization scale ambiguities will be rather large; for this reason we have evaluated the cross section for several plausible choices of the renormalization scale which leads to a rather broad spread in the cross section predictions. Given the CDF bounds, and choosing the scale which leads to the most conservation cross section, we find that the mass of the gluino, in the case where it fragments into a stable neutral hadron, must be in excess of $\simeq 170 \mathrm{GeV}$ from Run I data. For Run II we can make an estimate of the increased reach in gluino mass if no signal is observed 

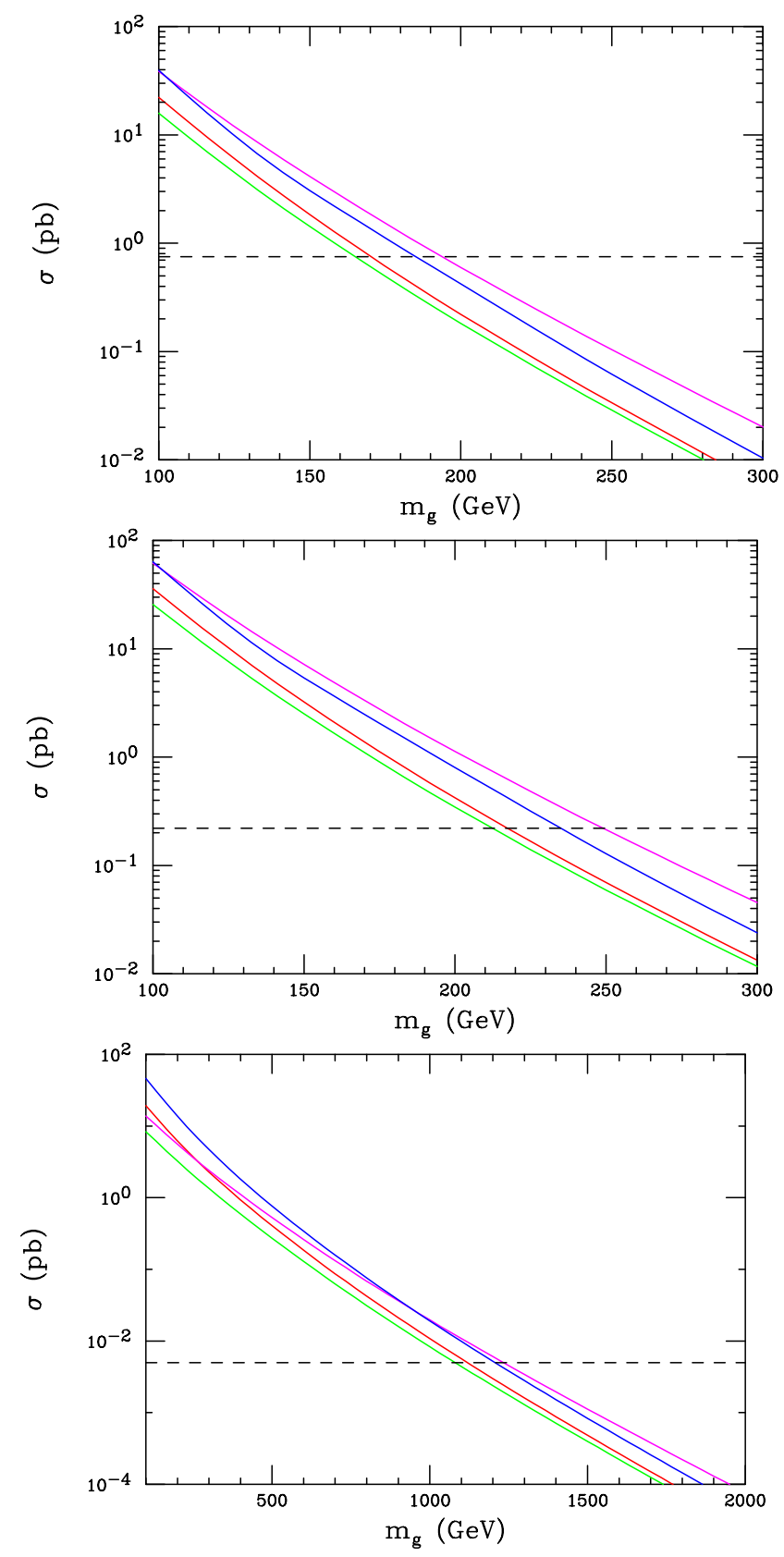

Figure 4: Total cross section for the production of $\tilde{g} \tilde{g}+$ jet at the Tevatron Run I (top), Run II (middle), and LHC (bottom). The cross section has been evaluated at four different renormalization scales (from top to bottom on the left side): the $p_{T}$ of the process (magenta), $m_{\tilde{g}}$ (blue), the invariant mass of the gluino pair (red), the center of mass energy of the parton level process (green).

above SM expectations, assuming identical detector requirements, by scaling by the square root of the integrated luminosity and allowing for the increase of the center of mass energy to $1.96 \mathrm{TeV}$. The results of this analysis are also shown in Fig. 4; here we see that gluino masses 
up to $\simeq 215 \mathrm{GeV}$ or more may be reached with an integrated luminosity of $1 \mathrm{fb}^{-1}$. Note that the scale uncertainties remain quite large. Looking further ahead, we can repeat this analysis for the LHC; here we will require the jet to have $E_{T} \geq 750 \mathrm{GeV}$ with an identical amount of missing energy to reduce SM backgrounds, and we will assume an integrated luminosity of $L=100 \mathrm{fb}^{-1}$. The SM backgrounds for this process at the LHC have been estimated by Vacavant and Hinchliffe [30] for the ATLAS detector within the context of searches for large extra dimensions. For the present analysis, we expect $\simeq 4200 \mathrm{SM}$ background events to pass our basic cuts. Our results are shown in Fig. 4 where we see that the gluino mass reach is now substantially improved to roughly $\simeq 1.1 \mathrm{TeV}$, assuming no excess is observed.

As is well-known, many new physics scenarios can lead to the monojet signature. If an excess of events is eventually found at either the Tevatron or LHC, is it possible to single out the production of neutral gluino hadrons as the source in any unique way? Here we must recall that while the passage of the neutral $R$-hadron through the detector will not be triggered on as a jet, the fact that a few hundred $\mathrm{MeV}$ of energy will be deposited every $\simeq 19 \mathrm{~cm}$ in small 'puffs' is a rather unique signature. The idea is then to examine the set of monojet events passing the cuts for additional hadronic activity corresponding to two 'tracks' of these 'puffs' left by the hadronic scattering of these neutral gluino hadrons.

Note that this analysis only depends on the fact that the $R$-hadron is a heavy, strongly interacting particle. Hence, the bounds derived above apply regardless of the hadronization pattern. These are thus (hadronization) model-independent bounds on the mass of the gluino. However, if the gluino does hadronize into charged states, the constraints may be significantly improved.

\subsection{Charged $R$-hadrons}

If the gluinos fragment predominantly into charged $R$-hadrons and they do not undergo charge exchange as they interact, it will be possible to observe them directly in the detector. In particular, their time delay (relative to a $\beta=1$ particle) [31] or their anomalously high ionization energy loss [32] as they cross the detector could be observed at the Tevatron. The bounds in this case will depend only on the production rate of gluino pairs without any additional bremsstrahlung. Recall that the $t$ - and $u$-channel diagrams mediated by squark exchange do not contribute in this model. In Fig. 5 we present the leading order cross section [19] at the renormalization scale $\mu=0.2 m_{\tilde{g}}$, which is the scale where the LO and NLO expressions match [33, 34].

The gluino pairs would be produced with a broad velocity distribution centered at values of $\beta$ that range from $\approx 0.7$ for $m=80 \mathrm{GeV}$ to $\approx 0.4$ for $m=500 \mathrm{GeV}$ (see Fig. 3). In particular, a significant fraction of them will be produced with a velocity below $\beta_{\max } \approx 0.65$. For these values of $\beta, \mathrm{CDF}$ and $\mathrm{D} 0$ can distinguish their time of flight from the tracking chambers to the muon chambers from that of a $\beta \approx 1$ particle [31]. In addition, energy loss will be dominated by ionization, which scales like $1 / \beta^{2}$. A charged hadron of mass $m \approx 500$ (200) $\mathrm{GeV}$ would be stopped inside the detector if its initial $\beta$ is below $\beta_{\text {min }} \approx 0.25$ (0.35) (see Section 3.1). Therefore, $R$-hadrons with velocity between $\beta_{\max }$ and $\beta_{\min }$ can be 
tracked all the way through the detector, and their time delay relative to a $\beta=1$ particle can be measured and correlated with the energy deposited in the hadronic calorimeter. For velocities up to $\beta=0.8$, just the determination of an anomalously high ionization energy loss (compared to a $\beta=1$ muon) is enough to establish bounds on the production rate of these charged $R$-hadrons.

The only published search for stable charged particles at the Tevatron based on time delay measures is from CDF, for a small integrated luminosity of $3.54 \mathrm{pb}^{-1}[31] .{ }^{1}$ The results of this search restrict the total cross section for pair production of stable particles with unit charge to be smaller than $\approx 15$ pb for $m=150 \mathrm{GeV}$ and $\approx 5$ pb for $m=500 \mathrm{GeV}$. In Fig. 5 we show that the gluino pair production cross section intersects the CDF bounds at $m \approx 200$ $\mathrm{GeV}$. Scaling by luminosity alone, we would expect the limit to increase to approximately $m_{\tilde{g}} \lesssim 270 \mathrm{GeV}$ based on time-of-flight measurements with $100 \mathrm{pb}^{-1}$ of integrated luminosity collected at the end of Run I. For the Tevatron Run II, with a luminosity of $2 \mathrm{fb}^{-1}$ this search technique is expected to cover the region $m_{\tilde{g}} \lesssim 430 \mathrm{GeV}[35]$. Note that this is a counting experiment with essentially no physics background. So, with enough luminosity the reach is simply limited by the center of mass energy of the collider, as seen from the expected Run II bound. Note also that the detector signal has a high dependence on the velocity of the produced stable particle, and gluinos produced near the kinematic limit will tend to have the same velocity, regardless of the energy of the collider. This allows us to estimate the reach at the LHC from time delay searches. Taking the limit on the number of events to be the same at the LHC as the Tevatron, we expect that an integrated luminosity of $100 \mathrm{fb}^{-1}$ at the LHC could explore the region $m_{\tilde{g}} \lesssim 2.4 \mathrm{TeV}$ (see Fig. 5).

CDF has also recently published constraints based on a measure of anomalous ionization, which they define as events with $d E / d x$ measurements high enough to correspond to $\beta \gamma \leq$ 0.85. Looking at Fig. 3, we see that this corresponds to gluino masses above roughly 100 $\mathrm{GeV}$. The high $d E / d x$ search technique suffers from backgrounds due to tracks for which the $d E / d x$ measurement fluctuated high or included extra ionization from an unreconstructed overlapping particle. For $\sqrt{s}=1.8 \mathrm{TeV}$ and a data sample of $90 \mathrm{pb}^{-1}$, CDF finds a limit of approximately $0.3 \mathrm{pb}$ for the production cross section of charged hadrons which do not undergo charge exchange; this bound is also displayed in Fig. 5. For the extreme case where the gluino always fragments into a charged hadron, this implies that its mass should be larger than $\approx 310 \mathrm{GeV}$. This bound is slightly higher than our expectations above for results from time-of-flight searches for heavy stable particles at the end of Run I. Due to the background considerations, it is problematic to scale these results for the LHC.

\subsection{Flippers}

An intriguing possibility is that the $R$-hadron can exchange charge with nuclei as it traverses the detector, so that the $R$ is "flipping" between being neutral and charged. This can occur if the $R$-hadron re-fragments after each hadronic interaction in the detector.

\footnotetext{
${ }^{1}$ We encourage CDF and D0 to update their results.
} 

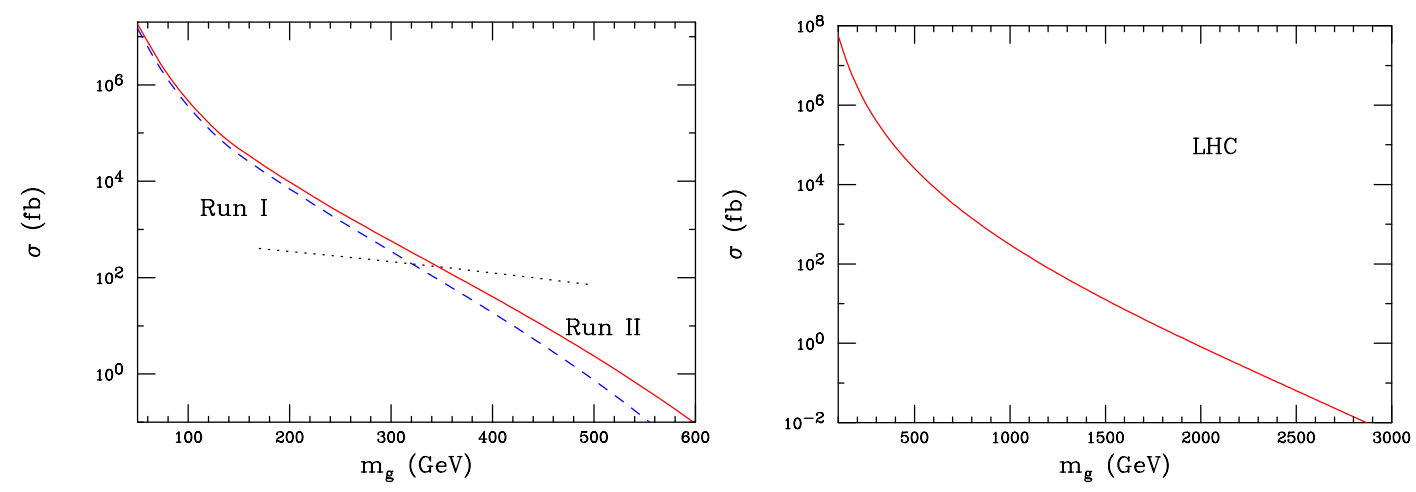

Figure 5: Total cross section for gluino pair production at the Tevatron (left) and LHC (right). These are evaluated at the renormalization scale $\mu=0.2 m_{\tilde{g}}$ suggested by the NLO calculation[33, 34].

This case can also be directly observed in the detector, with an efficiency that depends on the frequency of fragmentation into charged $R$-hadrons versus neutral $R$-hadrons. The probability rate for this charge exchange is clearly highly model dependent. Some models indicate that this charge exchange will happen if the hadronic interactions are dominated by Reggeon exchange, rather than the exchange of Pomerons or nuclear resonances [24], and if the mass splitting between the charged and neutral states is small. The most difficult signal occurs when both the produced $R$-hadrons remain neutral most of the time. In this case the bound obtained above via the monojet signature will apply. If the $R$-hadron is charged for a fraction of the time as it crosses the detector, then the bounds from the previous section will be somewhat weakened. However, it is difficult to estimate by how much given the large uncertainties inherent in modeling the fragmentation. The best one can say is that in the case of charge exchange, the constraints on the gluino mass is bounded by the searches in the two extreme cases discussed above and lies in the range $170-300 \mathrm{GeV}$ from the Tevatron Run I.

It may be possible to find flipper particles from an offline analysis of an excess monojet signal. As discussed above, if there is such an excess, it would be interesting to analyze the data set for a signal of the underlying physics. Charge exchange would result in charged tracks that stop and start again some distance away. The presence of these tracks in an excess monojet sample would be a spectacular signal of a heavy long-lived colored particle.

\section{Cosmic Rays}

Let us finally examine the possibility of observing the long-lived gluino in non-collider experiments. In particular, cosmic rays with energy above $500 \mathrm{TeV}$ could produce such particles when they strike a nucleon in the upper atmosphere. If that were the case, the $R$-hadrons could reach the detector at IceCube [36] and produce a distinct signature. Previous studies corresponding to $R$-hadrons being the source of super-GZK cosmic ray events can be found in $[37,38]$. 
To understand the type of signal that could be expected, let us consider an initial proton of energy $2 \times 10^{6} \mathrm{GeV}$ that hits an atmospheric nucleon (yielding a center-of-mass energy of $\sqrt{s}=2 \mathrm{TeV}$ ) and creates a pair of gluinos of mass $m_{\tilde{g}}=200 \mathrm{GeV}$. The typical invariant mass of the gluinos would be of order $500 \mathrm{GeV}$, with each gluino carrying a total energy $E_{0}=2.5 \times 10^{5} \mathrm{GeV}$ with (equal and opposite) transverse momenta of $100 \mathrm{GeV}$. The scattering angle between the two gluinos would be $\sim 10^{-3} \mathrm{rad}$.

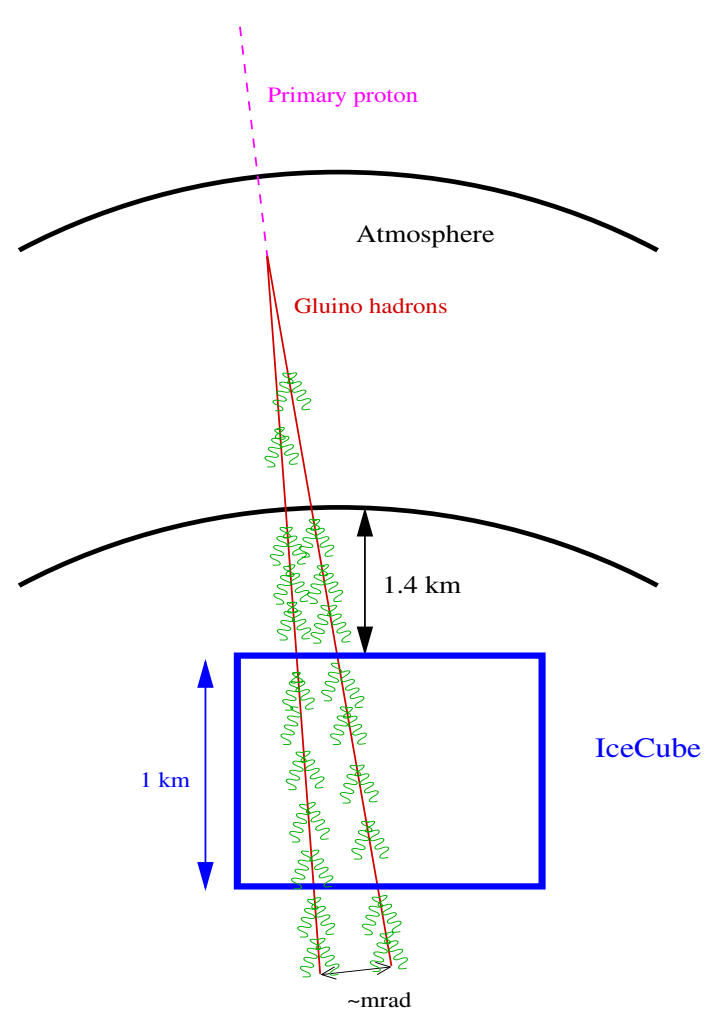

Figure 6: An illustration of the path of $R$ hadron pairs produced in the atmosphere and striking the IceCube detector.

Once hadronized, to reach the top of the IceCube detector the $R$-hadrons must cross the atmosphere (an approximate depth of $x_{a}=10^{3}$ $\mathrm{g} / \mathrm{cm}^{2}$ ) and $1.4 \mathrm{~km}$ of ice (a depth of $x_{1}=1.4 \times$ $\left.10^{5} \mathrm{~g} / \mathrm{cm}^{2}\right)$. In the first interaction with the atmospheric nucleons, each $R$-hadron will lose a total energy between $303 \mathrm{GeV}$ (case (1)) and $64 \mathrm{GeV}$ (case (2)). The two cases, described in section 3.1, represent two models describing the hadronic interactions of the heavy $R$-hadrons and represent two extreme scenarios. At these energies it is possible to approximate the average energy loss per interaction by $\Delta E=k \gamma$ with $k$ between $0.24 \mathrm{GeV}$ (case (1)) and 0.055 $\mathrm{GeV}$ (case (2)). For the interaction length in ice (as well as air) we take $\lambda_{T}(R)=(16 / 9) \lambda_{T}(\pi)$, with $\lambda_{T}(\pi) \approx 65 \mathrm{~g} / \mathrm{cm}^{2}$. In the linear approximation for the average energy deposited in each hadronic interaction, $\Delta E$, the energy of the $R$ hadron when it reaches a depth $x$ is

$$
E(x) \approx E_{0} e^{-\frac{k x}{m \lambda_{T}}}
$$

Therefore, in the atmosphere each $R$-hadron would deposit between 2.7 and $0.6 \mathrm{TeV}$. This energy would be homogeneously distributed, and the two jets would reach the ground separated by approximately $\sim 100 \mathrm{~m}$. It is unclear if such an anomalous profile could be detected in air shower experiments (note that this signal would come inside a $1.8 \times 10^{5} \mathrm{GeV}$ hadronic event).

The $R$-hadrons will then reach the top of the IceCube detector, which is $1.4 \mathrm{~m}$ below the Antarctic surface (see Fig. 6 for an illustration) each one with an energy $E\left(x_{1}\right)$ between 57 $\mathrm{TeV}$ (case (1)) and $178 \mathrm{TeV}$ (case (2)). The IceCube detector then extends to a depth of 2.4 $\mathrm{km}$. As the $R$-hadrons pass through the 1000 meters of ice $\left(\Delta x=10^{5} \mathrm{~g} / \mathrm{cm}^{2}\right)$, the two hadrons could be neutral or could be flipping charge between each hadronic interaction (approximately one per meter). Each $R$ would deposit a total energy around $37 \mathrm{TeV}$ (case (1)) or $38 \mathrm{TeV}$ (case (2)). It is amusing that both cases give a similar amount of total energy deposition in 
the detector: in case (1) $R$ deposits a larger fraction of energy per hadronic interaction, but it reaches IceCube with less energy than in case (2). If the hadron is predominantly charged there would be $\simeq 200 \mathrm{GeV}$ of extra energy deposited in the form of ionization. Although such a pair of $R$ hadrons, separated by 100 or 150 meters, would be detectable, a detailed simulation would be needed to see if they can be distinguished from a typical muon bundle in an air shower core.

We can now estimate how many of these events per year could be expected at IceCube. As a cosmic proton of energy $E$ enters the atmosphere, it will always interact with a nucleon. If the total cross section to produce a pair of gluinos is $\sigma_{p N}^{\tilde{g} \tilde{g}}$, a fraction $\sigma_{p N}^{\tilde{g} \tilde{g}} / \sigma_{p N}^{T o t}$ of these cosmic rays will produce the pair of $R$-hadrons. In our estimate we will take the approximation [39] of $\sigma(E)_{p N}^{T o t}=50 \mathrm{mb}$. For $\sigma_{p N}^{\tilde{g} \tilde{g}}$ we will use the partonic cross sections given in [19] with the scale $\mu=0.2 m_{\tilde{g}}$ suggested by the next to leading order calculation in [33, 34].

The flux of downgoing protons from zenith angles of $\cos \theta>0.5$ can be estimated as [39] $\mathrm{d} F / \mathrm{d} E=f_{1} E^{-2.7}$ (year $\left.\mathrm{km}^{2} \mathrm{GeV}\right)^{-1}$ for $E \leq 10^{7} \mathrm{GeV}$ and $\mathrm{d} F / \mathrm{d} E=f_{2} E^{-3.0}$ (year $\mathrm{km}^{2}$ $\mathrm{GeV})^{-1}$ for $E \geq 10^{7} \mathrm{GeV}$, where $f_{1} \approx 3.0 \times 10^{18}, f_{2} \approx 3.7 \times 10^{20}$, and $E$ is expressed in $\mathrm{GeV}$. In addition, we must consider the possibility that the gluino pair is created in a second (or later) collision, after the initial proton has already scattered off a nucleon and retained a fraction of its initial energy. To be definite, we will consider that at these energies the proton loses approximately $\sim 16 \%$ of its energy in each hadronic collision (we obtain this value from case (1) in [11]), and will neglect the possibility that the gluinos are produced by the scattering of secondary particles. This effect introduces an additional factor of $\sum_{i=0} 0.84^{1.7 i}=3.9$ in the flux at $E<10^{7} \mathrm{GeV}$ and a similar factor of 3.4 at $E>10^{7} \mathrm{GeV}$.

The number of events $/ \mathrm{yr} / \mathrm{km}^{2}$ can then be estimated as

$$
N=\int \frac{\mathrm{d} F}{\mathrm{~d} E} \frac{\sigma_{p N}^{\tilde{g} \tilde{g}}}{\sigma_{p N}^{T o t}} \mathrm{~d} E .
$$

In Fig. 7 we show the number of events that one may expect at the IceCube detector for different values of the gluino mass. For gluino masses above $170 \mathrm{GeV}$ we obtain $N<1$, although, of course, there would be a non-zero possibility to observe an isolated gluino pair event. Although this search reach is inferior to that from hadron colliders, it could serve as an independent cross-check of the hadron collider results.

\section{Summary and Conclusions}

In this paper we have examined the experimental signatures for the production of gluinos at colliders and in cosmic rays within the Split Supersymmetry scenario. Unlike in the MSSM, the gluinos in this scenario are relatively long-lived due to the large value of the squark masses which mediate their decay. Once this lifetime exceeds a few hundred nanoseconds the gluino becomes essentially stable as far as collider and cosmic ray detectors are concerned.

At colliders, gluinos fragment and form charged or neutral hadrons. It is possible that this fragmentation may prefer neutral or charged $R$-hadrons. These hadrons scatter several 


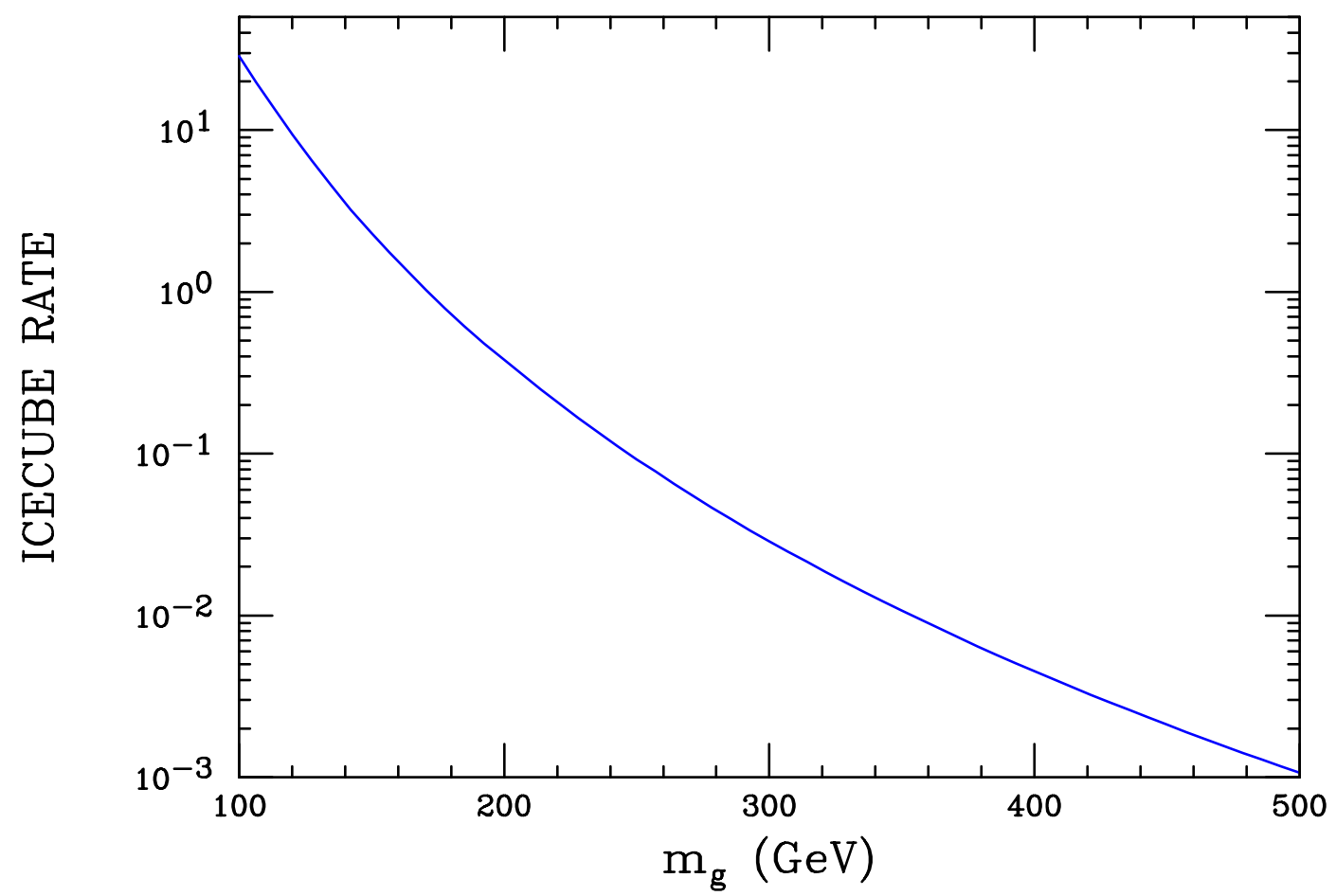

Figure 7: The number of double gluino events expected per year in IceCube.

times while passing through the detector but only deposit a small amount of energy during each hadronic interaction. In the limit where these hadrons are charged and remain so as they undergo scattering, they can be discovered through the conventional stable charged particle search. In this case the results from the Tevatron Run I place a lower limit on the gluino mass of $\simeq 310 \mathrm{GeV}$ which we expect to increase to $\simeq 430 \mathrm{GeV}$ for Run II provided no signal is observed. We can expect that the LHC will extend this into the TeV range. If the $R$-hadron's charge 'flips' during hadronic interactions in the detector, similar, though reduced, constraints are found. In the most difficult scenario to observe, the $R$-hadron is neutral and remains so as it traverses the detector. The soft energy deposition at each interaction is then seen to be too small to be triggered on or pass the cuts defining a jet, so that ordinary pair production of gluinos will not be observed. In order to have a reasonable trigger, we considered the production of a high- $p_{T}$ jet in association with the gluino pair that now appears as missing energy. Such a signature is common to many kinds of new physics scenarios. From Run I we found that a bound of $\simeq 170 \mathrm{GeV}$ can be placed on the gluino mass in this case, increasing to $\simeq 210 \mathrm{GeV}$ at Run II if no excess events are observed. For the LHC the corresponding reach is found to be $\simeq 1.1 \mathrm{TeV}$. Note that the $R$-hadrons will produce a monojet signal regardless of how they hadronize, so the monojet limit is completely model independent.

High energy protons in cosmic rays can collide with those in the atmosphere with sufficient center of mass energy as to produce gluino pairs which will have a small opening angle due to the large boost. Due to the small energy deposition in each interaction length, these 
meta-stable gluinos would be able to traverse the $\sim 1 \mathrm{~km}^{3}$ IceCube detector leaving a long string of energy depositions which could be observable above backgrounds. We estimate that the atmospheric gluino pair rate is sufficiently large that IceCube will be able to probe gluino masses up to $170 \mathrm{GeV}$. While this is inside the collider exclusion region, it is an important check in the case that there are unknown systematics or physics effects that mask the signatures in colliders.

We have shown that there exist robust constraints on the mass of the gluino in Split Supersymmetry. Additionally, much of the interesting region can be probed by the Tevatron Run II, and essentially all of the interesting region by the LHC.

\section{Note Added}

While this manuscript was in preparation, [40] appeared, where some signatures for long-lived gluino production at the LHC are also discussed.

\section{Acknowledgments}

The authors would like to thank Nima Arkani-Hamed, John Conway, Savas Dimopolous, Tom Gaisser, Francis Halzen, Mike Hildreth, Rich Partridge, Greg Landsberg, Enrique Zas, Chad Davis, and Peter Graham for helpful discussions.

\section{References}

[1] J. R. Ellis, Supersymmetry for Alp hikers, hep-ph/0203114. For a recent review, see this and references within.

[2] J. Ellis and D. Nanopoulos, Flavor changing neutral interactions in broken supersymmetric theories, Phys. Lett. B110 (1982) 44.

[3] J. Ellis, S. Ferrara, and D. Nanopoulos, CP violation and supersymmetry, Phys. Lett. B114 (1982) 231.

[4] J. Polchinski and M. Wise, The electric dipole moment of the neutron in low-energy supergravity, Phys. Lett. B125 (1983) 393.

[5] LEP Higgs Working Group Collaboration, Searches for the neutral Higgs bosons of the MSSM: Preliminary combined results using LEP data collected at energies up to 209-GeV, hep-ex/0107030.

[6] N. Arkani-Hamed and S. Dimopoulos, Supersymmetric unification without low energy supersymmetry and signatures for fine-tuning at the LHC, hep-th/0405159.

[7] G. F. Giudice and A. Romanino, Split supersymmetry, hep-ph/0406088.

[8] P. Kumar and J. D. Lykken, Visible sector supersymmetry breaking revisited, JHEP 07 (2004) 001, [hep-ph/0401140].

[9] H. Murayama and A. Pierce, Not even decoupling can save minimal supersymmetric SU(5), Phys. Rev. D65 (2002) 055009, [hep-ph/0108104]. 
[10] A. Arvanitaki, C. Davis, P. W. Graham, and J. G. Wacker, One loop predictions of the finely tuned SSM, hep-ph/0406034.

[11] H. Baer, K.-M. Cheung, and J. F. Gunion, A heavy gluino as the lightest supersymmetric particle, Phys. Rev. D59 (1999) 075002, [hep-ph/9806361].

[12] S. Raby and K. Tobe, The phenomenology of SUSY models with a gluino LSP, Nucl. Phys. B539 (1999) 3-22, [hep-ph/9807281].

[13] A. Mafi and S. Raby, An analysis of a heavy gluino LSP at CDF: The heavy gluino window, Phys. Rev. D62 (2000) 035003, [hep-ph/9912436].

[14] A. Pierce, Dark matter in the finely tuned minimal supersymmetric standard model, hep-ph/0406144.

[15] S.-H. Zhu, Chargino pair production at linear collider and split supersymmetry, hep-ph/0407072.

[16] B. Mukhopadhyaya and S. SenGupta, Sparticle spectrum and phenomenology in split supersymmetry: Some possibilities, hep-th/0407225.

[17] DELPHI Collaboration, J. Abdallah et. al., Search for an LSP gluino at LEP with the DELPHI detector, Eur. Phys. J. C26 (2003) 505-525, [hep-ex/0303024].

[18] ALEPH Collaboration, A. Heister et. al., Search for stable hadronizing squarks and gluinos in $e+e$ - collisions up to $s^{* *}(1 / 2)=209-G e V$, Eur. Phys. J. C31 (2003) 327-342, [hep-ex/0305071].

[19] S. Dawson, E. Eichten, and C. Quigg, Search for supersymmetric particles in hadron - hadron collisions, Phys. Rev. D31 (1985) 1581.

[20] P. F. Smith et. al., A search for anomalous hydrogen in enriched D-2 O, using a time-of-flight spectrometer, Nucl. Phys. B206 (1982) 333-348.

[21] M. L. Perl et. al., The search for stable, massive, elementary particles, Int. J. Mod. Phys. A16 (2001) 2137-2164, [hep-ex/0102033].

[22] G. R. Farrar and P. Fayet, Phenomenology of the production, decay, and detection of new hadronic states associated with supersymmetry, Phys. Lett. B76 (1978) 575-579.

[23] G. R. Farrar and P. Fayet, Bounds on $R$ hadron production from calorimetry experiments, Phys. Lett. B79 (1978) 442.

[24] A. C. Kraan, Interactions of heavy stable hadronizing particles, hep-ex/0404001.

[25] M. Moshe, Recent developments in Reggeon field theory, Phys. Rept. 37 (1978) 255-345.

[26] Z. Kunszt, E. Pietarinen, and E. Reya, Transverse momenta of hadronically produced heavy quark systems: $2 \rightarrow 3$ processes in QCD, Phys. Rev. D21 (1980) 733.

[27] J. F. Gunion and Z. Kunszt, Heavy flavor production with gluon bremsstrahlung, Phys. Lett. B178 (1986) 296-300.

[28] CDF Collaboration, D. Acosta, Search for Kaluza-Klein graviton emission in p anti-p collisions at $s^{* *}(1 / 2)=1.8-\mathrm{TeV}$ using the missing energy signature, Phys. Rev. Lett. 92 (2004) 121802, [hep-ex/0309051]. 
[29] Do Collaboration, V. M. Abazov et. al., Search for large extra dimensions in the monojet + missing- E(T) channel at D0, Phys. Rev. Lett. 90 (2003) 251802, [hep-ex/0302014].

[30] L. Vacavant and I. Hinchliffe, Model independent extra-dimension signatures with ATLAS, hep-ex/0005033.

[31] CDF Collaboration, F. Abe et. al., Limits on the production of massive stable charged particles, Phys. Rev. D46 (1992) 1889-1894.

[32] CDF Collaboration, D. Acosta et. al., Search for long-lived charged massive particles in anti-p p collisions at $s^{* * 1} 1 / 2=1.8-T e V$, Phys. Rev. Lett. 90 (2003) 131801, [hep-ex/0211064].

[33] M. Spira, Squark and gluino production at hadron colliders, hep-ph/9711408.

[34] W. Beenakker, R. Hopker, M. Spira, and P. M. Zerwas, Squark and gluino production at hadron colliders, Nucl. Phys. B492 (1997) 51-103, [hep-ph/9610490].

[35] D. Cutts and G. Landsberg, Detection of long-lived particles in Run II with D0, in Physics at Run II: Supersymmetry / Higgs Workshop (M. Carena and J. Lykken, eds.), 1998. FERMILAB-PUB-00-349.

[36] The IceCube Collaboration, C. Spiering et. al., The IceCube project, astro-ph/0404090.

[37] D. J. H. Chung, G. R. Farrar, and E. W. Kolb, Are ultrahigh energy cosmic rays signals of supersymmetry?, Phys. Rev. D57 (1998) 4606-4613, [astro-ph/9707036].

[38] V. Berezinsky, M. Kachelriess, and S. Ostapchenko, Extensive air showers from ultra high energy gluinos, Phys. Rev. D65 (2002) 083004, [astro-ph/0109026].

[39] Particle Data Group Collaboration, K. Hagiwara et. al., Review of particle physics, Phys. Rev. D66 (2002) 010001.

[40] W. Kilian, T. Plehn, P. Richardson, and E. Schmidt, Split supersymmetry at colliders, hep-ph/0408088. 